\title{
To scheme or to rule: Evidence against the Dual-Mechanism Model and the Rule-Based Learner
}

\author{
VSEVOLOD M. KAPATSINSKI \\ University of New Mexico
}

\section{Introduction}

The Dual Mechanism Model, or DMM, proposes that "regular" inflectional morphemes are attached by a categorical default rule understood as an operation over variables while "irregular" inflected words are formed by analogy to irregular forms in the lexicon (e.g. Pinker and Prince 1994).

The two defining characteristics of a regular pattern applied via a default rule are 1) the regular pattern is the most frequently used pattern with nonce stems that are not similar to any existing stems, i.e. it is the pattern that is applied by default when formation by analogy fails; and 2) the regular pattern is applies when certain necessary and sufficient conditions defining the variables to which the rule applies have been satisfied, e.g. the stem is a verb, thus the regular pattern should be applied as readily to nonce stems that are similar to no existing stems as to stems similar only to existing stems taking the regular pattern, i.e. the regular pattern does not exhibit a similarity effect.

A serious challenge to DMM has come from Albright and Hayes (2003) who found that even the regular English past tense exhibits a similarity effect. Thus when native English speakers are asked to rate regular and irregular past tense forms of nonce verbs on how natural they sound as the past tense of the verb, they rate regular forms higher if the verb is similar to many regular verbs and no irregular ones than if it is similar to neither regulars nor irregulars. These results suggest that no pattern is free from similarity effects.

However, the DMM can account for these effects. Pinker and Prince (1994) stated that high frequency inflected forms are stored in the lexicon, whether they are regular or irregular. There is no theoretical mechanism that would prevent speakers from forming analogies based on these stored regulars. The analogical mechanism would then apply to all patterns but the rule would be a mechanism that is only used to attach regular patterns. Under this account, the default pattern does not have to be free of similarity effects but must simply be less susceptible to similarity effects than its competitor patterns. 
A stronger case against the DMM would be made if morphological domains in which the pattern that is least susceptible to similarity effects is not the default pattern or multiple patterns are equally productive and sensitive to the contents of the neighborhood of nonce stimuli could be found.

Dabrowska (2001) argued that there is no default singular genitive in Polish because all singular suffixes were frequently overgeneralized by children, as opposed to one suffix being overgeneralized much more than the others, like in the past tense domain in English. However, the rate of overgeneralization is not an unambiguous indicato of high productivity in the sense in which it is used within DMM: high productivity for the proponents of the DMM means high rates of application to nonce stimuli that are similar to no words in the speakers' lexicons because this is the context in which no analogical models are present, hence the default has to be used. Rates of overgeneralization do not allow us to distinguish among productivity in sparse and dense neighborhoods, hence the findings of Dabrowska (2001) could be argued to result from examining contexts where analogical formation is possible.

Kapatsinski (2005) has argued that Russian verbal ste extensions are a domain in which the most productive suffix is not the suffix that exhibits the smallest similarity effect. He presented native Russian speakers with nonce monosyllabic nouns and asked them to form verbal infinitives. The subjects most often suffixed -a- or - $\mathrm{i}$ - to the noun to form the infinitive. The experiment included stimuli similar to existing verbal roots taking -a-, those similar to verbal roots taking -iand those similar to no existing roots. The difference in how productive -a- was with stimuli similar to -a-taking roots and stimuli similar to no existing roots was significantly smaller than the difference in how productive -i- was with stimuli similar to -i-taking roots and stimuli similar to no existing roots. Thus, -aexhibited lower sensitivity to the contents of the nonce stimulus's neighborhood than - $\mathrm{i}$ - did in the study. Nonetheless, -i- was more productive than -a-with coronal- and palatal-final stimuli similar to no existing roots, despite -aexhibiting lower sensitivity to the contents of the stimulus' neighborhood for the same stimuli. Thus, the defining characteristics of the default were shown to be dissociable, contra the DMM.

Possible objections to Kapatsinski (2005) are:

1) There was no guarantee that there was the same degree of similarity between stimuli similar to -i-bearing words and their -i-bearing neighbors as between stimuli similar to -a-bearing verbs and their -abearing neighbors. If stimuli that had many -i-bearing phonological neighbors were more similar to their neighbors than stimuli with -abearing neighbors were to theirs, -a- may well be more sensitive to similarity to -i- or -a- and -i- may be equal in sensitivity to similarity 
and there would still be a greater similarity effect for -i- than for -abecause -i- has a greater difference in similarity to be sensitive to. ${ }^{1}$

2) Given that the noun-final consonant was shown to influence the relative productivity of -a- and -i- (Kapatsinski 2004, 2005b), the fact that the identity of the noun-final consonant was not controlled across experimental conditions (similar to -i-, similar to -a-, similar to none) could make stimuli similar to no existing roots more similar to stimuli similar to -a- bearing roots than to stimuli similar to -i-bearing roots.

In this paper, we present an experiment in which similarity and final consonant identity are tightly controlled. The experiment uses an empirically grounded similarity metric and a systematic method of similarity estimation.

In addition, instead of asking the subjects to come up with a verb given a noun, they were presented with noun-verb pairings and asked to rate how likely people are to form the verb from the noun on a 10-point scale. Albright and Hayes (2003) have previously found that for the English past tense ratings obtained in this task are highly correlated with the probability of producing the past tense in an elicited production task. Both -i- and -a- turned out to be sensitive to the contents of the nonce stimulus's neighborhood and, as long as the identity of the root-final consonant is controlled, sensitive to the same degree. Thus, the results provide evidence that there is no single default among the Russian stem extensions. Type frequency and sublexical morphophonological associations explain the results.

Finally, while the earlier experiment used visual presentation of stimuli, auditory stimulus presentation is used in the present study.

\section{Methods}

\subsection{The Fixed Radius Method (FIRM) of controlling for similarity}

It is necessary to take into account all words that are close enough to the nonce stimulus to affect its assignment to one of the inflectional classes, i.e. all words in the stimulus' neighborhood (Luce and Pisoni 1998) and to weigh the influence of each of these words by its proximity to the stimulus so that more similar words influence category assignment more.

The method used in this study may be termed The Fixed Radius Method, or FIRM (Kapatsinski 2005, 2005b). Under FIRM, the experimenter first makes up a nonce stimulus, chooses a radius of $\mathrm{X}$ units of phonological similarity and finds all words that are within that radius of the stimulus. In this paper, a reverse dictionary of Russian (Zaliznjak 1977), and the Ogonek Corpus of modern written discourse collected in the 1990's (SFB441, 2000) were searched to obtain words in the neighborhood of a given nonce stimulus.

\footnotetext{
${ }^{1}$ This is a potential problem with all studies of sensitivity to similarity conducted so far, including those that provide support for the DMM, except, perhaps, Albright and Hayes (2003) because of the lack of empirically grounded similarity metrics.
} 
Second, the experimenter derives a similarity score for each of the words, which is the inverse of the number of units of similarity (UNOS) by which the word differs from the nonce stimulus. The similarity score of each of the inflectional classes in the domain is the sum of the similarity scores of its member words which are within the neighborhood radius of the stimulus. The class with the highest similarity score is the class that the stimulus is closest to. Stimuli that are not close to any existing words have no neighbors within the radius.

FIRM makes two assumptions. One is that the neighborhood is a discrete set, i.e. the set of words that are brought to bear on a word's category assignment can be delimited (cf. Luce and Pisoni 1998). The second is that the neighborhood has a graded internal structure, i.e. that distance from the stimulus within the neighborhood, rather than simple membership or non-membership in the neighborhood is relevant.

\subsection{The similarity metric}

The UNit Of Similarity (UNOS) was based on the Halle (1995) feature.

Feature changes implied by more specific changes were counted, e.g. the change from [d] to [n] involves changing the features [nasal] and [sonorant] but the change in sonority is implied by the change in nasality. This was done because of the finding, reported by McInish and Tikofsky (1969) and Derwing and Nearey (1986) that number of mismatched features is correlated with perceived similarity of English CVC stimuli that differ by one consonant. The change from one value to another of the non-binary feature [place] was always counted as 1 UNOS, regardless of the distance between values in articulatory space.

A mismatch in X UNOS distributed over two consonants differed in 1.1X UNOS. The coefficient of 1.1 was empirically derived as the average of the ratios in the two conditions in Experiment B of Kapatsinski (2005b). In this experiment, native English speakers were presented with nonce monosyllabic stimuli that differed in either one consonant or two contiguous consonants. The location of the mismatch was controlled and so was the type of mismatch in terms of the features by which the stimuli differed. The temporal duration of the match was also controlled. The conditions differed in whether the temporal duration of the mismatch was the same, regardless of the number of mismatched segments or the difference in temporal duration between one mismatched segment and two mismatched segments was even larger than in naturalistic stimuli.

Substitutions of allophones of the same phoneme ${ }^{2}$ were counted as changes by $1 / 2$ UNOS, as were substitutions of one phoneme for another in environments where they would be realized in the same way phonetically in all experiments. For example, voiced obstruents devoice at the ends of words in Russian, hence the phonemic contrast between /t/ and /d/ is neutralized in this position and the distance between $/ \mathrm{kot} /$ and $/ \mathrm{kod} /$ is half a unit. This assumption awaits empirical testing.

\footnotetext{
${ }^{2}$ Resulting from substituting adjacent phonemes
} 
A mismatch in X UNOS in the final position was counted as 1.2X UNOS, the coefficient being derived from experiment A of Kapatsinski (2005c), a sound similarity judgment task with bisyllabic nonsense words presented to native English speakers. All words differed by two consonants where either both consonants were medial, one medial and one initial, one medial and one final, or one initial and one final. Because experiment A used bisyllabic stimuli, the relevance of the coefficient to CVC stimuli used in the experiment reported here not apparent. However, the same coefficient is found in Bendrien's (1992) results with nonsense CVC stimuli.

Finally, a mismatch in X UNOS that was distributed across the onset and the rhyme was counted as 1.4X UNOS, the coefficient being derived from experiment A of Kapatsinski (2005b). Either the penalty for cross-segment distribution or for cross-constituent distribution was applied to a given pair of stimuli.

The radius in all experiments was 4 UNOS (based on Connine et al. 1993, who found that monosyllabic words that differ by 4 features do not prime each other in phonological priming). All stimuli shared at least one segment. Number of segments was reflected in the temporal durations of match and mismatch. ${ }^{3}$

A control set of words that are not similar to any of the existing words was derived for each experiment. Such a control set is necessary to evaluate the productivity of various patterns and its dependence on similarity (Clahsen 1999). According to the DMM, the pattern used most in this set of stimuli is the default pattern. With the FIRM, this is a set of words that do not have any neighbors within the radius or whose neighbors from different classes are equal in number and equidistant from the stimulus.

Clearly, more work is needed to determine that the similarity metric's assumptions hold for Russian. However, we have reasons to suspect that they would hold, at least qualitatively: 1) final mismatches are more salient than initial mismatches even in non-linguistic stimuli (cf. Kidd and Watson 1992 for tone sequences, Coble and Robinson 1992 for white noise), except for body languages (Yoon and Derwing 2001), which Russian is not, and in Russian final consonants have much influence on the productivity of the stem extensions, 2) mismatches involving longer sounds are more salient outside of English as well (see Tamariz 2005 for Spanish, Coble and Robinson 1992, Kidd and Watson 1992 for nonlinguistic sounds), and 3) discontinuous mismatches are more salient than continuous mismatches in nonlinguistic sounds (cf. Coble and Robinson 1992, Kidd and Watson 1992). Thus, most features of the metric are neither languagenor Language-specific.

\footnotetext{
${ }^{3}$ This control is necessary because Kapatsinski (2005c, experiment A) found that the same words are perceived to sound less similar if their mismatched consonants are artificially lengthened, while remaining phonologically the same.
} 


\subsection{Participants and Task}

Eighteen native Russian speakers were presented auditorily with a nonsense noun followed by a verb formed from it via either -i-, -a-, -ova-, or -eva-. They were asked to rate the pairs on a 10 point scale ranging from 1="nobody would form this verb from this noun" to $10=$ "everyone would form this exact verb from this noun”. Subjects were asked to imagine that the nouns are recent borrowings and they need a verb to describe an event involving the noun.

\subsection{Stimuli}

Minimal pairs of nouns similar to no existing nouns were created by varying the place of articulation of the final consonant. Thus, there were minimal pairs exemplifying contrasts between $/ \mathrm{b} /$ and $/ \mathrm{d} /$, between $/ \mathrm{p} /$ and $/ \mathrm{t} /$, between $/ \mathrm{v} /, / \mathrm{z} /$, and $/ \mathbf{z} /$, and between $/ \mathrm{m} /$ and $/ \mathrm{n} /$. Subjects' reactions to the members of the minimal pairs were compared to determine the influence of the root-final consonant independently of the influence of the neighbors and the non-final segments. Velar consonants were not used so as not to confound the necessity to perform an extra operation with the identity of the consonant. All consonants were non-palatalized when occurring noun-finally.

Stimuli similar to roots taking -a- and -i- were created so that the set of stimuli similar to -a-bearing words, the set of stimuli similar to -i-bearing words and the comparison set of stimuli similar to no existing words contained the same proportions of final consonant types. Thus, the sets of stimuli similar to -i-bearing and -a-bearing words each contained two nouns ending in $/ \mathrm{b} /, 1$ ending in $/ \mathrm{m} /, 1$ ending in $/ \mathrm{t} /$, and 1 ending in $/ \mathrm{p} /$. The set of stimuli similar to no existing words was twice as large, containing $4 / \mathrm{b} /$-final and $2 / \mathrm{p} /$-final, $/ \mathrm{m} /$-final, and /t/-final nouns. Thus any differences found between the sets cannot be attributed to the influence of the root-final consonants.

All stimuli similar to -a-taking words had no -i-taking words within the radius and vice versa. This was done to ensure that the magnitude of the differences between -a-favoring and -i-favoring words vs. neutral words are not due to an experimental confound but are rather a genuine reflection of differences in -a- and -i-'s sensitivity to the contents of the nonce stimulus's neighborhood. We also controlled the distance from the stimulus to the nearest neighbor.

Stress was assigned to the first syllable to half of the stimuli similar to no existing verbs and to the second syllable for the other half. It was assigned in a way that maximized similarity for the stimuli similar to -a-bearing and -i-bearing verbs. Stimuli are presented in table 1.

Table 1. Stimuli used to test the size of similarity effects or -a- and $-\mathbf{i}^{-}{ }^{4}$

\begin{tabular}{|c|c|c|}
$\begin{array}{c}\text { Stimuli similar to } \\
\text {-a-bearing words }\end{array}$ & $\begin{array}{c}\text { Stimuli similar to } \\
\text {-i-bearing words }\end{array}$ & $\begin{array}{c}\text { Stimuli similar to no } \\
\text { words }\end{array}$ \\
\hline
\end{tabular}

\footnotetext{
${ }^{4}$ The stimuli are presented in a phonological IPA transcription.
} 


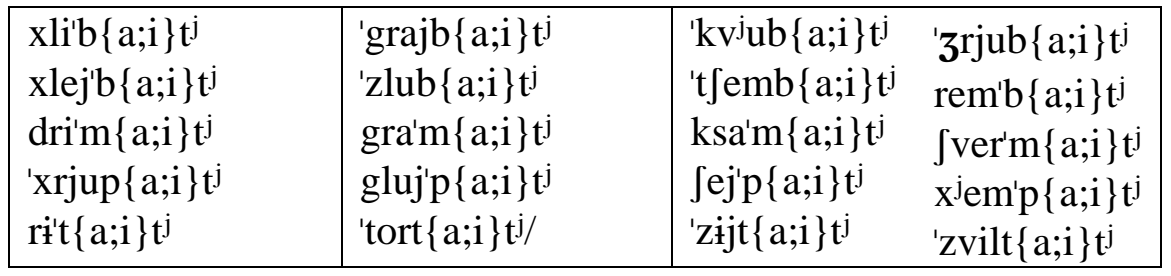

\subsection{Procedure}

Stimuli were presented in a pseudorandomized order using a random sequence from random.org. Locations in a second random sequence were used to assign positions to the first of two members of a minimal pair or two stimuli similar to words taking the same stem extension that were assigned by the first sequence to be within 10 stimuli of each other. In addition, it was ensured that the first 10 stimuli were similar to no existing words and that they were followed by one stimulus similar to -a- and one similar to -i-. The order of the -a-favoring and -ifavoring stimuli was switched for half of the subjects. There was no significant difference between the resulting subject groups.

Each stimulus was presented once. Subjects were tested in groups. One female native Russian speaker pronounced all of the stimuli for all the subjects. The subjects were not presented with orthographic representations of the stimuli. The scales were drawn on response sheets.

\subsection{Analysis}

The data were analyzed using a univariate ANalysis Of VAriance (ANOVA) in SPSS. Respondent identity was entered into the ANOVA as an independent variable. In addition, all stimuli were coded for stress location since half of the stimuli similar to no existing words were stressed on the root and half were stressed on the affix while stress location in stimuli similar to -a-bearing or -ibearing words was chosen to maximize the number of same-category members.

\section{Productivity and sensitivity to similarity}

As Table 2. shows, both -a- and -i- show robust similarity effects. Moreover, the sizes of the effects seem to be relatively equal. If this is indeed the case, we are forced to conclude that the more even lexical distribution of -a- makes it harder to find similarity effects for -a- methodologically but does not make -a-'s application less reliant on the contents of the stimulus's neighborhood. 
Table 2. Acceptability of nonce verbs bearing -a- and -i- depending on the contents of their phonological neighborhoods

\begin{tabular}{|l|c|c|}
\hline $\begin{array}{l}\text { The stimulus bears } \rightarrow \\
\text { Neighbors bear }\end{array}$ & A & I \\
\hline A & 6.27 & 5.16 \\
\hline I & 4.73 & 6.40 \\
\hline No neighbors & 4.70 & 5.05 \\
\hline Significance & $\mathrm{p}<0.0005$ & $\mathrm{p}<0.0005$ \\
\hline $\begin{array}{l}\text { Size of the similarity effect } \\
\text { =A/No Neighbors }\end{array}$ & 1.33 & 1.27 \\
\hline
\end{tabular}

However, while the final consonant has been controlled in the dataset explored in table 2, some stimuli similar to -a- or -i- are 0.5 UNOS removed from the nearest neighbor and some are 1 UNOS removed. A central prediction of the FIRM is that the internal structure of the neighborhood is graded. That is, distance from the stimulus within the neighborhood influences the similarity of the neighbor to the stimulus, as opposed to all neighbors within the radius being equally similar to the stimulus. Stimuli similar to -a- contain 3 stimuli 0.5 UNOS removed from their a-bearing neighbors, while stimuli similar to -i- contain only 1 stimulus 0.5 UNOS removed from its -i-bearing neigbor. Therefore, we may be overestimating -a-'s similarity effect in relation to -i-.

Table 3 presents the mean scores for stimuli similar to -i- and -a- and stimuli similar to no existing roots as a function of the distance between the stimulus and the nearest possible analogical model. At every distance from the nearest analogial model, - $\mathrm{i}$ - is the more productive of the two affixes. However, the difference is only significant when the nearest neighbor is 0.5 UNOS away $(p=0.009)$. These results suggest that $-a-$ may be slightly more sensitive to similarity than -i-, as found in experiment I. However, only one of the stimuli similar to -i- has a neighbor within 0.5 UNOS. Thus the data sample is small and we should be cautious in making this inference. Whether -a- is slightly more sensitive to similarity than -i- or not, it is clear that -i- is not more sensitive to similarity than -a- and is not less productive than -a-. Thus, the findings directly contradict the Dual Mechanism Model. 
Table 3. Distance to nearest neighbor and sensitivity to similarity

\begin{tabular}{|l|l|c|c|}
\hline $\begin{array}{l}\text { Similar to } \\
\text { verbs bearing }\end{array}$ & $\begin{array}{l}\text { Distance to } \\
\text { neighbor }\end{array}$ & -a-bearing stimuli & -i-bearing stimuli \\
\hline -a- & 0.5 & $\begin{array}{c}6.89 \\
\text { dif. from 1: } \mathrm{p}=.005\end{array}$ & 5.13 \\
\cline { 2 - 4 } & 1 & $\begin{array}{c}5.65 \\
\text { dif. from none: } \mathrm{p}=.005\end{array}$ & 5.19 \\
\hline -i- & 0.5 & 5.28 & $\begin{array}{c}8.22 \\
\text { dif. from 1: } \mathrm{p}<.0005\end{array}$ \\
\hline & 1 & 4.56 & $\begin{array}{c}5.89 \\
\text { dif.from none: } \\
\mathrm{p}=.011\end{array}$ \\
\hline None & & & 5.05 \\
\hline Similarity effect (0.5/None) & 1.70 & 1.6 \\
\hline Similarity effect (1/None) & 1.2 & 1.2 \\
\hline
\end{tabular}

Because 3 stimuli labeled as similar to verbs bearing -a- are acoustically identical to real verbs bearing -a-, and 1 stimulus similar to -i-taking verbs is acoustically identical to an -i-taking verb, one could hypothesize that the similarity effects observed are simply due to these verbs being perceived as real verbs bearing -i- and not due to analogy at all. If this were the case, there would be no difference between -a-bearing stimuli similar to -a-taking verbs for which the nearest neighbor is 1 UNOS away and -a-bearing stimuli similar to verbs taking -i-. Similarly, there should be no difference between -a-bearing stimuli similar to -a-taking verbs for which the nearest neighbor is 1 UNOS away and -abearing stimuli similar to verbs taking -i-. However, for both -a- and -i-bearing verbs, there is a significant difference between stimuli 1 UNOS removed from a model and those with no model within 4 UNOS (Table 3). Thus the results do indicate that similar verbs, and not just identical ones, are taken into account.

Since -i- is not less productive than -a- and is not more sensitive to similarity, the DMM is forced to hypothesize that - $\mathrm{i}$ - and -a- must be less productive than some other stem extension, which must be less sensitive to similarity than both of them. The only plausible alternative is -ova-/-eva-, since the stem extension is reasonably frequent and is often applied to borrowings. Table 4 shows the relative naturalness ratings of stimuli derived from the same set of nouns similar to no existing words with -i-, -a-, -ova-, and -eva-. Contrary to the Dual Mechanism Model's prediction, -i- and -a- are the stem extensions that derive the most natural-sounding verbs. The lower acceptability of -ova-/-evabearing words may be due to their greater length (cf. Frisch et al. 2000 for nonsense words in English). The acceptability of -a- and -i-bearing stimuli may also be artificially inflated by presenting subjects with stimuli similar to -i- and a-bearing verbs but not to -ova- and -eva-bearing verbs. However, the same result is obtained with a standard elicited production task (Kapatsinski 2005). The difference between -a- and -ova-/-eva- is highly statistically significant 
$(p<0.0005)$ when assessed in an ANOVA that also includes respondent identity, stress location, and final consonant identity. The difference between -i- and -a- is also significant when tested in the same way $(p=0.008)$. Nonetheless, the similarity effects displayed by -i- and -a- are relatively equal. The difference between -ova- and -eva- is not significant $(\mathrm{p}=0.564)$.

Table 5. Naturalness ratings of (coronal- and palatal-final) verbs derived from nouns similar to no existing words with -a-, -i-, -ova-, or -eva-.

\begin{tabular}{|l|c|c|c|c|}
\hline Stem extension & $-\mathrm{i}-$ & -a- & -ova- & -eva- \\
\hline Mean rating & 5.28 & 4.97 & 4.32 & 4.30 \\
\hline
\end{tabular}

\section{Response competition}

Tables 3 and 4 show that verbs bearing -a- are just as acceptable when they are similar to -i-bearing verbs as they are when they are similar to no existing words. Similarly, high similarity to -a-bearing verbs and lack of similarity to any existing verbs have the same effect on -i-bearing verbs. This suggests that the differences in judgments are not due to lower phonotactic probabilities of words that are similar to no existing words. In addition, it appears that the judgment is based solely on the characteristics of words in the stimulus's neighborhood that bear the stem extension the stimulus bears, rather than on the characteristics of all words in the stimulus's neighborhood: stimuli similar to words bearing stem extensions other than the one borne by the stimulus are similar to stimuli similar to no words. On the other hand, stimuli that are similar to the 'wrong' stem extension might be expected to reduce the acceptability of the stimulus if stem extensions are able to compete. Despite the instructions, which explicitly mentioned estimating the likelihood of producing this exact verb given the noun, subjects seem to have based their judgments solely on the activation level of the stem extension borne by the stimulus and not on the activation levels of other extensions.

Lack of response competition in the data presents problems for connectionist models using distributed representation, since the degree of attractiveness of an output is inversely proportional to the degree of attractiveness of all other outputs in the model because outputs are represented as alternative parameter settings over the same set of nodes. Localist models, in which different outputs are represented by separate nodes or sets of nodes do not suffer from this problem.

\section{Determinants of productivity in "default" circumstances}

One objection might be that even though we have shown that the characteristics of the default are dissociable, we have not eliminated the need for a default. That is, we have not proposed an alternative account of what happens when no analogical models are in the neighborhood of the stimulus.

First, let us note that in both the experiment reported here and in the nonce probe elicited production task reported in Kapatsinski (2005), the most productive stem extensions are the stem extensions with the highest type frequency: -i-and - 
a- each account for about a third of the types in Zaliznjak (1977), supporting the idea that type frequency influences productivity (Bybee 1995).

The relative productivity of -i- and -a- with different roots similar to no existing roots also depends on how frequently parts of the stimulus's root cooccur with -i- as opposed to -a-.

Kapatsinski (2005b, chapter 7) has shown that native Russian speakers judge nonce verbs bearing -i- to be more natural in the same task as used in the experiment reported here if the body (onset+nucleus) of the root frequently occurs in verbs bearing -i- than if it frequently occurs in verbs bearing -a- when overall body frequency is controlled. Similarly, verbs bearing -a-are judged to be more natural if their body co-occurs with -a- than when their body co-occurs with -i-.

Finally, a separate group of stimuli, which were similar to no existing roots and comprised minimal pairs differing only in the identity of the root-final consonant was presented to subjects as part of the experiment described above. Final consonant identity had several reliable effects. Thus, -a-bearing verbs were more acceptable if their roots ended in /d/ than if their roots ended in /b/ (mean scores were 5.19 and 4.82 respectively, $\mathrm{p}=0.030$ ), if their roots ended in $/ \mathbf{3} /$ than if their roots ended in $/ \mathrm{v} /(5.11$ versus $4.50, \mathrm{p}=0.040)$. Verbs bearing -eva- were more acceptable if their roots ended in $/ \mathrm{m} /$ than when their rootsended in $/ \mathrm{n} / \mathrm{(4.78}$ vs. 4.12, $\mathrm{p}=0.040$ ). The suffix -i- was more acceptable after $/ \mathrm{z} /$ than after $/ \mathrm{v} /$ or $/ \mathbf{3} /$ (5.42 vs. 4.68, $\mathrm{p}=0.019$ ). The suffix -a- was also more acceptable after $/ \mathrm{z} /$ than after $/ \mathrm{v} /(5.23$ vs. $4.50, \mathrm{p}=0.015)$. Thus, it is clear that phonological composition of the root has an effect on productivity that extends beyond determining the composition of the neighborhood. Sublexical phonological units seem to be able to form associations with different suffixes and thus increase the resting activation levels of the associated morphemes when perceived.

\section{Conclusion}

In this paper, we have replicated the finding that the stem extensions with the highest type frequency are the most productive stem extensions in Russian using a

new task and an auditory mode of presentation, as predicted by Network Theory (Bybee 1995, 2001). Furthermore, we have shown that the stem extensions are approximately equal in productivity and in sensitivity to the contents of the nonce stimulus's neighborhood, indicating that there is no single default stem extension, contrary to the Dual Mechanism Model (Pinker and Prince 1994). We have shown that sublexical phonological units can form associations with suffixes and influence their productivity beyond the influence of the contents of the stimulus's neighborhood and can account for differences between stimuli similar to no existing words, which are left without explanation in the Dual Mechanism Model. Finally, we presented evidence that environments favoring an output do not necessarily disfavor other outputs, suggesting that a localist implementation of Network Theory (e.g. LAST, Kapatsinski 2005b, forthcoming) is more appropriate than a distributed connectionist one. 


\section{References:}

Albright, A., and B. Hayes. 2003. Rules vs. analogy in English past tenses: A computational/experimental study. Cognition, 90 (1), 119-61.

Bendrien, T. A. 1992. Sound similarity judgments in English CVC's. BA Honors Thesis: U of Alberta.

Bybee, J. L. 1995. Regular morphology and the lexicon. Language and Cognitive Processes, 10, 425-55.

Bybee, J. L. 2001. Phonology and language use. Cambridge: Cambridge University Press.

Coble, S. F., and D. E. Robinson. 1992. Discriminability of bursts of reproducible noise.Journal of the Acoustical Society of America, 92, 2630-5.

Connine, C. M., D. G. Blasko, and D. Titone. 1993. Do the beginnings of spoken words have a special status in auditory word recognition? Journal of Memory and Language 32: 193-210.

Dabrowska, E. 2001. Learning a morphological system without a default: the Polish genitive. Journal of Child Language, 28, 545-74.

Derwing, B. L., and T. M. Nearey. 1986. Experimental phonology at the University of Alberta. In J, Ohala, ed. Experimental phonology, 187-209. San Diego: Academic Press.

Frisch, S. A., N. R. Large, and D. B. Pisoni. 2000. Perception of wordlikeness: Effects of segment probability and length on the processing of nonwords. Journal of Memory and Language, 42, 481-96.

Kapatsinski, V. M. forthcoming. Frequency, neighborhood density, age-ofacquisition, and lexicon size effects in priming, recognition and associative learning: Towards a single-mechanism account. Proceedings of HDLS VI, Albuquerque, NM.

Kapatsinski, V. M. 2005. Characteristics of a rule-based default are dissociable: Evidence against the Dual Mechanism Model. In S. Franks, F. Y. Gladney, and M. Tasseva-Kurktchieva, eds. Formal approaches to Slavic linguistics 13: The South Carolina meeting, 136-46. Ann Arbor, MI: Michigan Slavic Publications.

Kapatsinski, V. M. 2005b. Productivity of Russian stem extensions: Evidence for and a formalization of Network Theory. M.A. Thesis: Universityof New Mexico.

Kapatsinski, V. M. 2004. Type frequency vs. phonotactic probability as determinants of Russian verbal stem extension productivity. Poster presented at the $4^{\text {th }}$ International Conference on the Mental Lexicon, Windsor, ON.

Kapatsinski, V. M. 2004b. Measuring phonological similarity of English syllables. Ms. Available at http://www.unm.edu/ alator/PhoneticSimilarity.pdf (Forthcoming in proceedings of the $1^{\text {st }}$ UGA Linguistics Society Graduate Student Conference).

Kidd, G. R., and C. S. Watson. 1992. The "proportion-of-the-total-duration rule" for the discrimination of auditory patterns. Journal of the Acoustical Society 
of America, 92, 3109-18.

Luce, P. A., and D. B. Pisoni. 1998. Recognizing spoken words: The neighborhood activation model. Ear and Hearing, 19, 1-36.

McInish, J. R., and R. S. Tikofsky. 1969. Distinctive features and response latency: A pilot study. Perception and Psychophysics, 6, 267-8.

SFB 441, Project B1. 2000. Ogonek 1996-2000. http://heckel.sfb.unituebingen.de/cgi-bin/cqp.pl

Tamariz, M. 2005. Exploring the adaptive structure of the mental lexicon. Ph.D. Thesis, U of Edinburgh.

Yoon, Y. B., and B. L. Derwing. 2001. A language without a rhyme: Syllable structure experiments in Korean. Canadian Journal of Linguistics, 46 (3/4), 187-237.

Zaliznjak, A. A. 1977. Grammaticheskij slovar' russkogo jazyka: Slovoizmenenie. Moscow: Russkij Jazyk. 